\title{
High dynamic range and high dispersion stability \\ of a magnetorheological grease damper for semi-active vibration suppression
}

\author{
Authors \\ Toshihiko SHIRAISHI*, Shuto NAGAMATSU and Hirotaka MISAKI (*corresponding author) \\ E-mail \\ Toshihiko SHIRAISHI (shira@ynu.ac.jp)
}

\begin{abstract}
Affiliation
Graduate School of Environment and Information Sciences, Yokohama National University

(79-7 Tokiwadai, Hodogaya-ku, Yokohama, 240-8501, JP)
\end{abstract}

\begin{abstract}
In this study, the dynamic range and dispersion stability of a shear-type magnetorheological (MR) grease damper were experimentally investigated as important characteristics affecting the performance of semi-active vibration suppression. Furthermore, the damper was applied to the semi-active vibration suppression of a small single-degree-of-freedom model structure subjected to seismic excitation. The performance test results of the damper indicated that it has a dynamic range of 157 times, which is much higher than that of conventional MR dampers. Also, because of the high dispersion stability of MR grease, the results showed that its performance can be kept for 9 days longer in comparison with a similar damper using MR fluid. Moreover, the structural vibration suppression test results demonstrated that the damper can suppress vibration response over a wide frequency range by applying skyhook-based control, which can take full advantage of the damper's high dynamic range.
\end{abstract}

\section{Keywords}

Magnetorheological grease, semi-active vibration suppression, dynamic range, dispersion stability

1. Introduction

Semi-active vibration suppression is a method of suppressing vibrations by varying the stiffness or damping characteristics of a damper according to the vibration state of a system. When a controllable damper is used for semi-active vibration suppression, to generate a desired force and realize high suppression performance, the damping force should be changed quickly, widely, and continuously. Dampers that use magnetorheological (MR) fluids have been widely studied and applied in many applications, as they satisfy the requirements for controlling the damping force of dampers. MR fluid is a suspension of micron-sized magnetic particles dispersed in a carrier fluid, and this method was first reported in a fluid clutch application (Rabinow, 1948). When an external magnetic field is applied to MR fluid, its rheological characteristics are rapidly changed. The magnetization of the particles in the fluid results in the formation of chain-like clusters along the applied field. In this phenomenon, which is 
called the MR effect, the MR fluid reversibly changes from a liquid state to a semi-solid state in milliseconds (Dyke et al., 1998). Such change can be activated by an electric current, as the current can be converted into a magnetic field in an electromagnetic coil. In a semi-active vibration suppression system using an MR damper, variable damping characteristics are achieved by supplying a variable current to an electromagnetic coil in the damper. As MR dampers can achieve high-performance suppression by just controlling the current, they have received a lot of attention and have been frequently studied for many applications, including civil engineering structures and vehicle suspension systems (Lee et al., 2010; Park et al., 2010; Tu et al., 2011; Yao et al., 2002).

However, conventional MR fluid dampers have two issues to be resolved. The first issue is the sedimentation of their magnetic particles due to the density mismatch between the particles and their carrier oil. In structural applications, when a damper is exposed to a static state for a long time, sedimentation causes the damping force to diverge from the desired force, resulting in performance degradation. The second issue is the low dynamic range due to the sliding friction force acting on the sealing elements of dampers, where the dynamic range is the ratio of the total force to the uncontrollable force, and it is one of the most important parameters for evaluating the performance of MR dampers (Yang et al., 2002). This sliding friction increases the uncontrollable force and reduces the dynamic range of MR dampers. However, sealing elements are essential for confining the MR fluid in dampers. To achieve both high dispersion stability of magnetic particles and high dynamic range of the damper, other materials such as elastomer, plastomer, and grease have been proposed as better carriers of magnetic particles (Kintz et al., 2003; Shen et al., 2004; Xu et al., 2011). While MR elastomer has a problem of low MR effect, MR plastomer and MR grease can offer both high dispersion stability and high MR effect, thus they have promising usage in the field of vibration suppression (Ahamed et al., 2018). However, few applications to controllable dampers have been reported before despite their potential. For high reliability and high suppression performance in practical applications, particles should be prevented from settling and the sliding friction force acting on sealing elements should be eliminated.

To overcome these issues, a shear-type damper using magnetorheological grease (a shear-type MR grease damper) was developed (Shiraishi and Sugiyama, 2015). It has an oscillating plate moving in a box filled with MR grease (Figure 1), which is mainly composed of micron-sized magnetic particles and grease as a dispersion medium. The MR grease used in the damper was developed by researchers including one of the authors of this paper (Kimura et al., 2013). This damper worked subject to the following mechanism. First, a magnetic field was induced inside the electromagnetic coil by supplied currents. Then, a closed magnetic circuit was formed through the iron core. The generated magnetic field passed through the MR grease perpendicular to the plate oscillation. In the gap between the oscillating plate and the inner wall of the box, magnetic particles of the MR grease formed chain-like clusters along the field, which resisted shear flows. The damping force value of the shear-type MR grease damper was obtained as the product of the damper plate area and the shear stress of the MR grease that was determined by a magnetic flux density and shear rate. Therefore, by determining the plate area, the winding number of the coil, and electric currents considering the size of the target structure, the damper can be applied to seismic control.

The combination of the developed MR grease and shear-type damper could solve the abovementioned two issues. The sedimentation issue could be mitigated by the MR grease, and high dispersion stability was realized. 
The low dynamic range issue could be overcome by the shear-type damper without the need for sealing elements. Unlike MR fluids, semi-solid MR grease does not leak out during operation, so it can be applied to open structures when the internal pressure is not used to generate forces. As the sliding friction force of shear-type MR grease dampers is theoretically zero, the dynamic range is high. Furthermore, the absence of sealing elements enhances the reliability by reducing the number of mechanical parts. Thus, high dispersion stability and high dynamic range can be achieved for MR dampers using MR grease in combination with shear-type dampers. However, this method needed to be experimentally and quantitatively verified in detail.

In this study, two experiments using the shear-type MR grease damper were conducted. A performance test of the damper was performed to investigate its two advantages: high dynamic range of the damper and high dispersion stability of the MR grease. Then, a structural vibration suppression test was conducted to investigate the semiactive vibration suppression performance of a structure equipped with the damper. In this experiment, the displacement response of the upper floor of a small single-degree-of-freedom model structure subjected to actual seismic excitation was evaluated as a performance indicator.

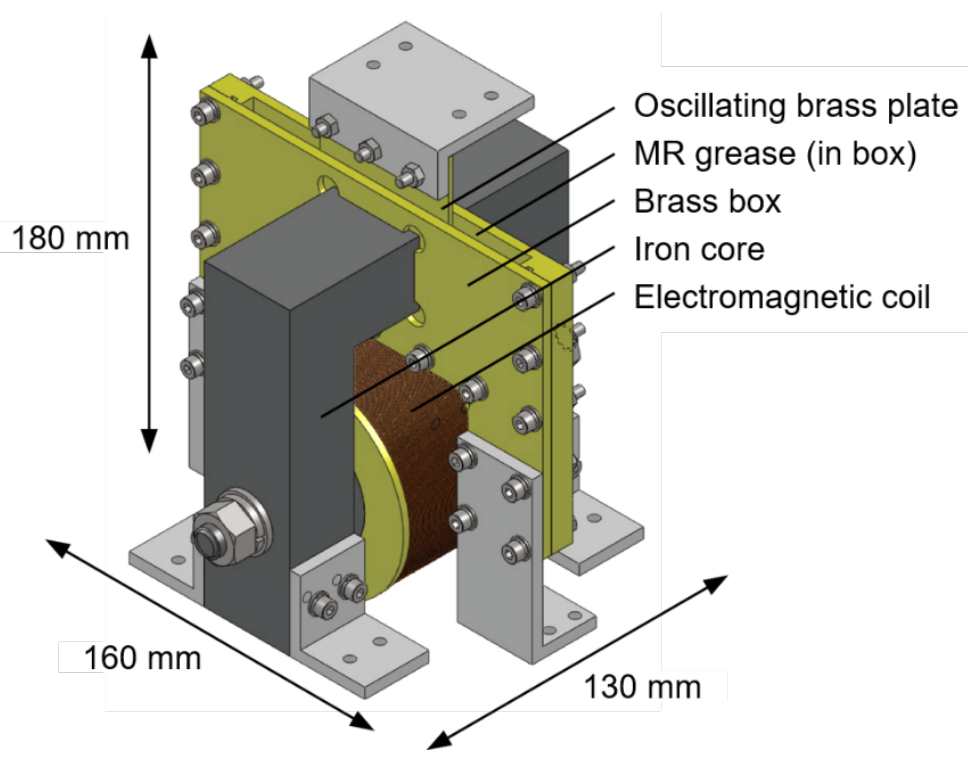

(a)

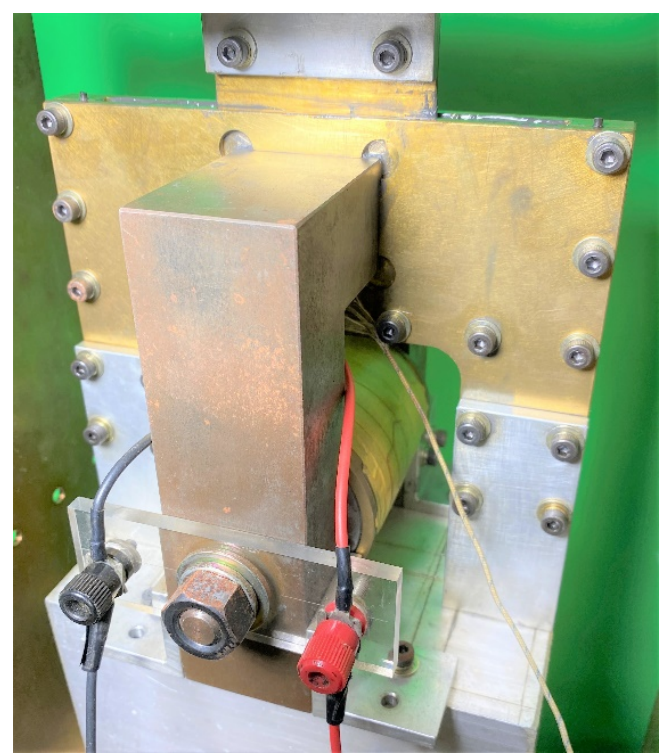

(b)

Figure 1. (a) Schematic and (b) photograph of the shear-type MR grease damper. 
2. Magnetorheological grease

Magnetorheological grease is a suspension of magnetic particles in grease, and it was developed to mitigate the sedimentation of MR fluid particles (Kintz et al., 2003; Rankin et al., 1999). After its development, several studies have focused on its application in controllable devices (Chansheng, 2006; Kavlicoglu et al., 2013) and on measuring its rheological characteristics (Bahiuddin et al., 2019; Mohamad et al., 2019; Wang et al., 2019). However, to ensure that MR grease can perform well as a working fluid in controllable dampers, its response time and dispersion stability should also be experimentally investigated. The characteristics of the MR grease used in this study had already been examined. It has been experimentally confirmed that the maximum shear stress of the MR grease and the time constant of the damping force of a prototype damper using the MR grease are as high as those of conventional dampers using MR fluids (Shiraishi et al., 2011) (Figure 2). Furthermore, the dispersion stability of the MR grease has been investigated through static observations (Kimura et al., 2013; Shiraishi et al., 2011; Shiraishi and Misaki, 2016).

In this study, a static observation was performed for 50 days to examine the dispersion stability of conventional MR fluids and that of the MR grease, and their particle concentrations were found to be 82 and $75 \mathrm{wt} \%$, respectively (Table 1). Also, their dispersion stabilities were quantified using the sedimentation ratio, which was defined in a previous study as the ratio of the volume of the supernatant liquid in a fluid to that of the entire suspension (Tu et al., 2011).

The developed MR grease remained evenly dispersed. However, the conventional MR fluid was separated into two layers because of sedimentation (Figure 3). In the static state, the MR fluid produced a visible supernatant with a volume fraction of $20 \%$ in total for 50 days (Figure 4). This result indicates that the performance of the dampers that use MR fluids decreases when they are applied to actual structures, as they are exposed to a static state for a long time and then subjected to sudden disturbances. In the MR grease, no visible supernatants were observed, even after a 50 day static state (Figure 4). This improvement is attributed to the capacity of grease to suspend particles. By focusing on the inner structure of the MR grease, the particles may be held using a thickener, which forms threedimensional network structures in grease. These results indicate that dampers using the MR grease can realize highperformance structural vibration suppression for a longer time compared with conventional MR fluid dampers, as the high dispersion stability of the MR grease allows its dampers to generate desired forces even after a static state.

In the subsequent section, the effect of the high dispersion stability of MR grease on the damping force was described. 


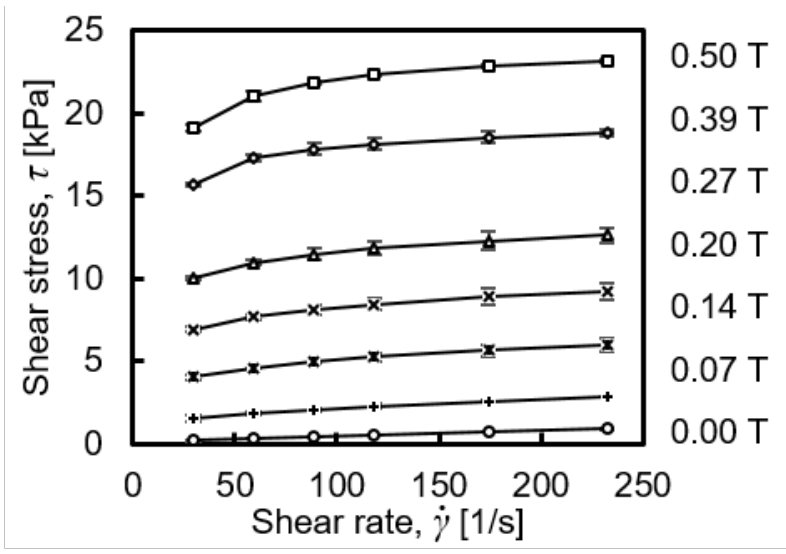

Figure 2. Relationship between the shear stress and shear rate of the developed MR grease in the case of particle concentration of $75 \mathrm{wt} \%$ (Shiraishi et al., 2011).

Table 1. Specifications of conventional MR fluid and the developed MR grease.

\begin{tabular}{ccc}
\hline & MR fluid & MR grease \\
\hline Magnetic particle & Fe & Carbonyl iron \\
Particle concentration & $82 \mathrm{wt} \%$ & $75 \mathrm{wt} \%$ \\
Base oil & Hydrocarbon & Mineral oil \\
Thickener & - & Lithium soap \\
Thickener concentration & - & $1 \mathrm{wt} \%$ \\
\hline
\end{tabular}
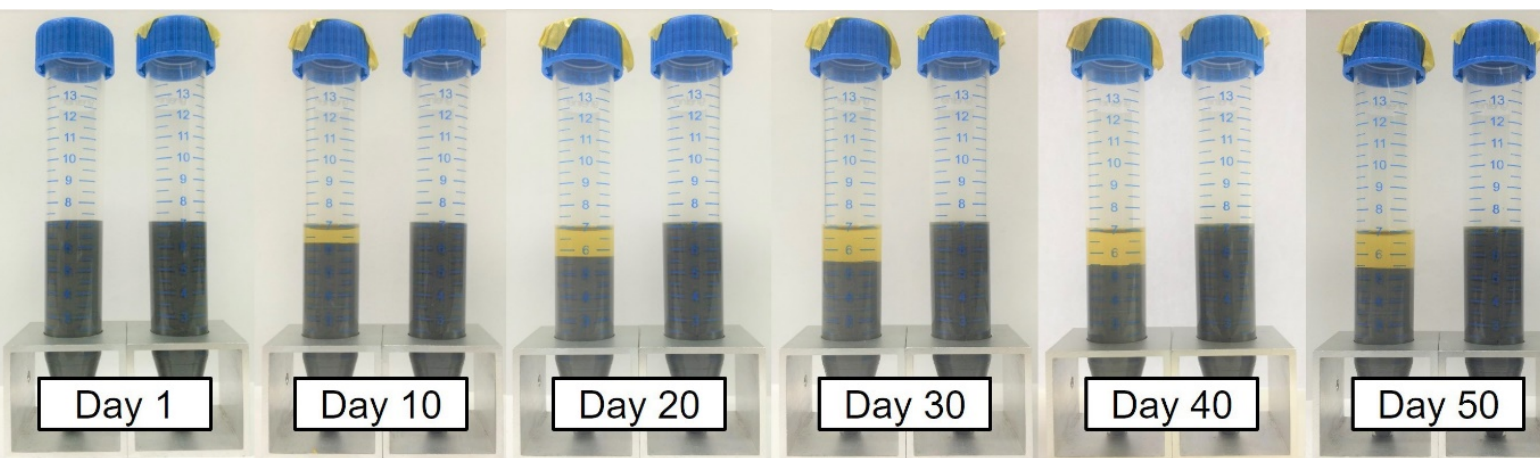

Figure 3. Static observation of conventional MR fluid (left) and the developed MR grease (right).

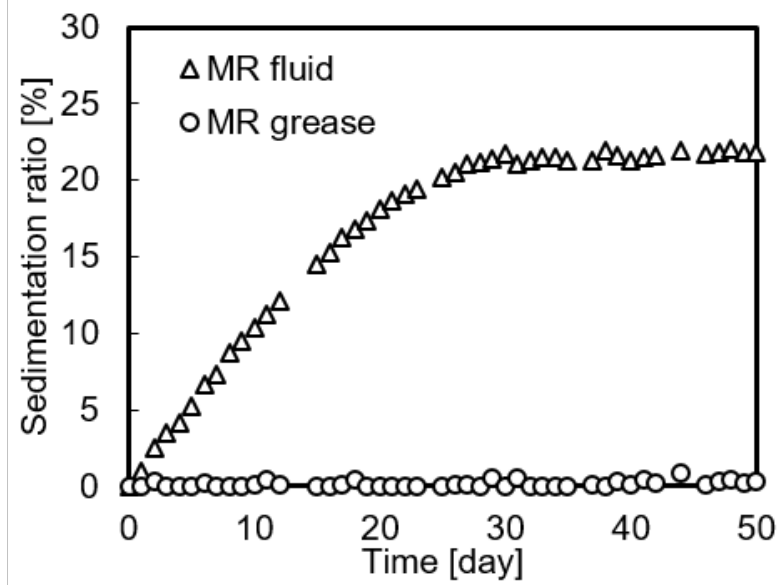

Figure 4. Time series of the sedimentation ratio of conventional MR fluid and the developed MR grease. 
3. Performance test of the shear-type MR grease damper

To investigate the dynamic range and dispersion stability of the shear-type MR grease damper, the relationship between its damping force and damper plate displacement was obtained during the excitation of the damper plate (Figure 5). The force and displacement were measured using a load cell and a laser displacement sensor, respectively. The damping characteristics of the damper were varied by varying an electric current from a bipolar power supply to the electromagnetic coil in the damper. The force and displacement were measured for each current by setting the current to a constant value. In this section, the two advantages of the shear-type MR grease damper were demonstrated. First, the high dynamic range of the MR grease damper was described as a relationship between the damping force and velocity. Second, the high dispersion stability of the MR grease was described as a time variation of the damping force.

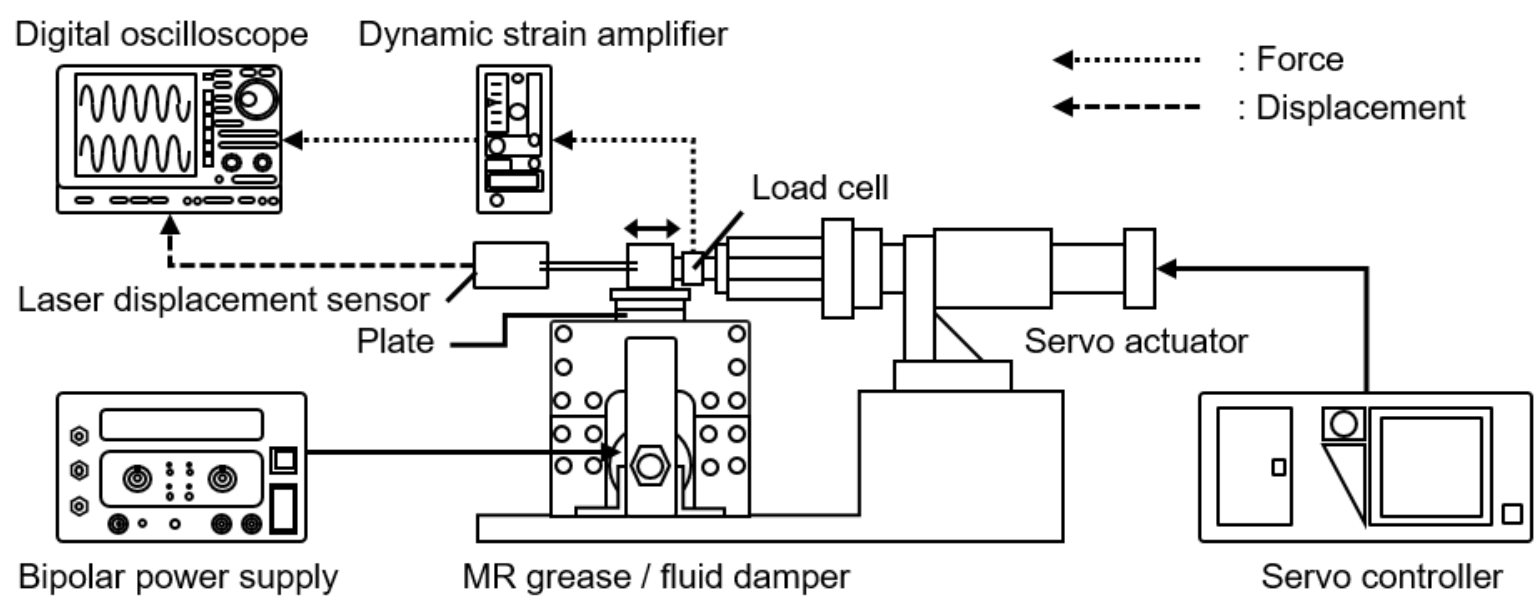

Figure 5. Schematic diagram of the experimental setup for the performance test of the MR damper. 
3.1 Dynamic range of the MR grease damper

\section{Methods}

To obtain the dynamic range, which is the ratio of the force in the ON-state to that in the OFF-state, the damping force was measured at the current values of $0,1,2,3$, and $4 \mathrm{~A}$ (Table 2). The maximum current of $4 \mathrm{~A}$ was determined based on the wire temperature. Note that it had been confirmed by simulations and experiments that the magnetic flux density linearly increased with the increase in the supplied current until it reached $0.5 \mathrm{~T}$ at $4 \mathrm{~A}$. The damper plate was excited using a sine wave with a constant displacement amplitude of $5 \mathrm{~mm}$. To examine the velocity effect on the dynamic ranges, the experiment was conducted at 1,3 , and $5 \mathrm{~Hz}$. The damper plate velocity was obtained as a time derivative of the displacement measured using the sensor. That velocity was called the "damper velocity" below in this paper. The test temperature of $40^{\circ} \mathrm{C}$ was chosen to conduct experiments with a constant temperature of the MR grease. Considering that the supplied current heated the electromagnetic coil and raised the temperature of the MR grease, it was necessary to set the test temperature to a higher value than the room temperature.

Table 2. Experimental conditions for measuring the dynamic range of the shear-type MR grease damper.

\begin{tabular}{cc}
\hline Current & $0,1,2,3,4 \mathrm{~A}$ \\
Displacement amplitude & $5 \mathrm{~mm}$ \\
Frequency & $1,3,5 \mathrm{~Hz}$ \\
Winding number of the coil & 1000 turns \\
Temperature & $40^{\circ} \mathrm{C}$ \\
\hline
\end{tabular}




\section{Results and discussion}

The damping force increased with the increase in the supplied current and damper velocity. The current effect on the damping force was more dominant than the damper velocity effect (Figure 6). A force-current diagram was obtained by plotting the maximum damping force at each current. The damping force almost linearly increased with the increase in the supplied current at all the frequencies (Figure 7). The dynamic range at each frequency was calculated as the ratio of the maximum force at $4 \mathrm{~A}$ to that at $0 \mathrm{~A}$. In the case of $1 \mathrm{~Hz}$, a dynamic range of 157 times was obtained as the ratio of 94.7 to $0.6 \mathrm{~N}$. This experimental value corresponded with the theoretical one calculated using the shear stress of the MR grease. Similarly, dynamic ranges of 125 and 110 times were obtained at 3 and $5 \mathrm{~Hz}$, respectively.

As far as we surveyed, the dynamic range values of the shear-type MR grease damper were much higher than those of conventional MR dampers (Table 3). If an MR fluid were applied to a shear-type damper, a high dynamic range could be obtained due to its low viscosity in the OFF-state. However, in practical applications, an MR fluid cannot be used as the working fluid of a damper without sealing elements since it may leak out during operation. On the other hand, the semi-solid MR grease, which allowed the sealing elements to be removed, contributed to an extremely high dynamic range of over 100 times, which has never been achieved before. Moreover, the elimination of the sealing elements also improved the damper reliability as the number of mechanical parts decreased.

Compared with a previous study (Shiraishi and Misaki, 2016), the dynamic range of the shear-type MR grease damper was increased. In this study, the MR grease amount was decreased to a minimum to just cover the crosssection of the iron core in the ON-state based on the attractive force acting on the MR grease by the magnetic field. In this condition, the damper could keep a large damping force in the $\mathrm{ON}$-state and minimize the damping force in the OFF-state.

These results indicate that the shear-type MR grease damper has the capability of successfully suppressing vibrations, as its high dynamic range allows it to generate the required damping forces. 


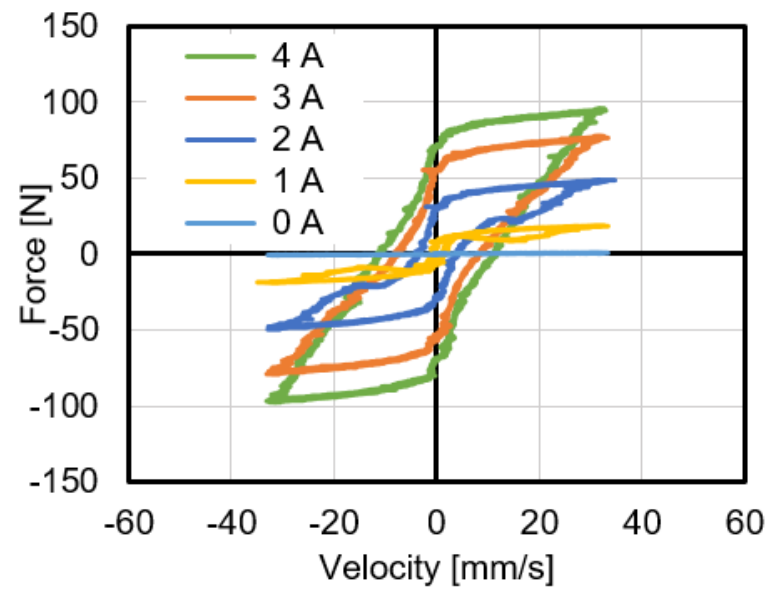

(a)

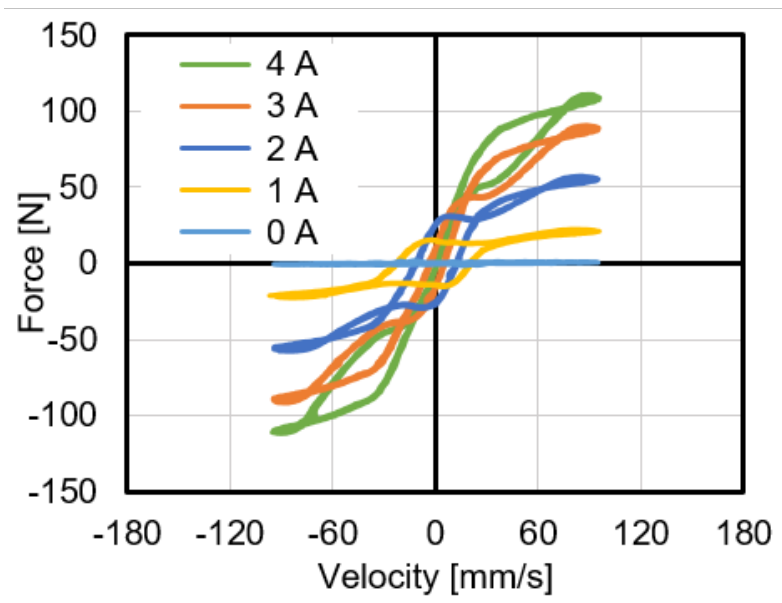

(b)

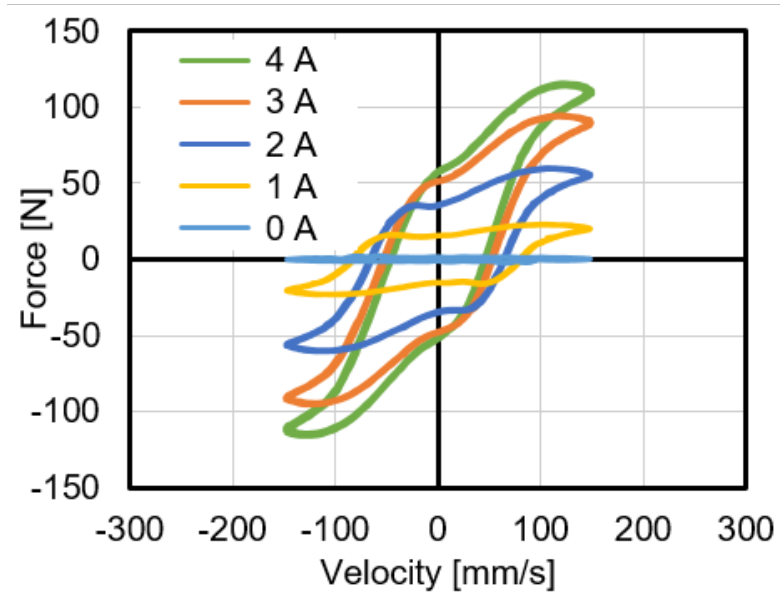

(c)

Figure 6. Relationship between the damping force and damper velocity at (a) $1 \mathrm{~Hz}$, (b) $3 \mathrm{~Hz}$, and (c) $5 \mathrm{~Hz}$. 


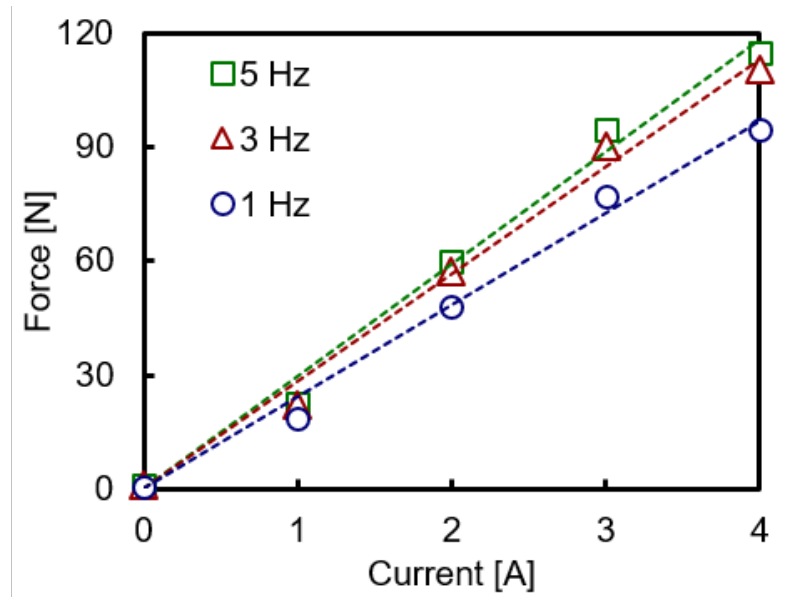

Figure 7. Relationship between the maximum damping force and current. The forces were approximated as linear current functions (dotted line).

Table 3. Experimental dynamic ranges of conventional MR dampers and the shear-type MR grease damper.

\begin{tabular}{lc}
\hline \multicolumn{1}{c}{ Damper type } & Dynamic range \\
\hline Frictionless MR fluid damper (Machacek et al., 2019) & 9 \\
Pressure-type MR fluid damper (Yang et al., 2002) & 10 \\
Pressure-type MR fluid damper (Tu et al., 2011) & 18 \\
Shear-type MR grease damper in the present study & 157 \\
\hline
\end{tabular}

3.2 Dispersion stability of the MR grease in the damper

\section{Methods}

The damping force was measured before the static period and 9 days after it to investigate the dispersion stability effect on the damping force (Table 4). The developed MR grease and a conventional MR fluid were used as working fluids for the shear-type damper. It was examined whether the high dispersion stability of the MR grease contributed to keeping the damper's damping force for a long time or not by comparing the forces of the shear-type damper in the cases of using the MR grease and MR fluid. The force was measured when starting to move the damper just after stirring and 9 days after the static state, as the particle sedimentation would degrade the performance. The damper plate was excited by a sine wave with a displacement amplitude of $10 \mathrm{~mm}$ and a frequency of $1 \mathrm{~Hz}$. The currents of 0 and 4 A were supplied to the electromagnetic coil, which had 125 turns. These conditions were set considering a small structure for the vibration suppression tests described below.

Table 4. Experimental conditions for investigating the dispersion stability in the MR damper.

\begin{tabular}{cc}
\hline Static period & 9 days \\
Displacement amplitude & $10 \mathrm{~mm}$ \\
Frequency & $1 \mathrm{~Hz}$ \\
Current & $0 \mathrm{~A}(\mathrm{OFF}), 4 \mathrm{~A}(\mathrm{ON})$ \\
Winding number of the coil & 125 turns \\
Temperature & $40^{\circ} \mathrm{C}$ \\
\hline
\end{tabular}




\section{Results and discussion}

In the OFF-state with the current of $0 \mathrm{~A}$, the damping force of the MR grease damper was kept for 9 days, whereas that of the MR fluid damper significantly increased (Figure 8). However, in the ON-state with the current of 4 A, both the MR grease and fluid dampers kept their damping forces (Figure 9). Regarding the change in the root mean square (RMS), the damping force was evaluated $10 \mathrm{~s}$ after the beginning of excitation. The MR grease damper generated almost the same damping force in the OFF and ON states, even 9 days after the static state. However, the damping force of the MR fluid damper increased by $41.6 \%$ in the OFF-state (Table 5). The redispersibility of the MR fluid was so low that it took more than $1 \mathrm{~h}$ to return to the desired performance.

Considering the static observation results, the produced supernatants, which had a volume fraction of $10 \%$, led to the performance degradation of the MR fluid damper 9 days after the static state. In the OFF-state, the dense particles around the bottom of the box increased the viscosity of the MR fluid and caused an undesired large damping force. In the ON-state, the damping force of the MR fluid damper could be kept by the magnetic attractive forces. According to the visual observation of the MR fluid surface, the magnetic particles became supernatants and fully covered the cross-section of the iron core as soon as the magnetic field was applied. The same experiment was conducted at the lower current of $1 \mathrm{~A}$ to examine the field strength effect, and it was found that the damping force generated on day 10 corresponded to that generated on day 1 although the attractive force was smaller than that at $4 \mathrm{~A}$. These findings indicate that the MR fluid sedimentation affected the performance in the OFF-state 9 days after the static state.

These results also indicate that the high dispersion stability of the MR grease allows its dampers to generate desired damping forces both in the OFF and ON states. When the damper was applied to the semi-active vibration suppression of a structure, the vibration response could be successfully suppressed for a long time, even after the static state as the damper could generate a force corresponding to the desired force calculated based on the control law. 


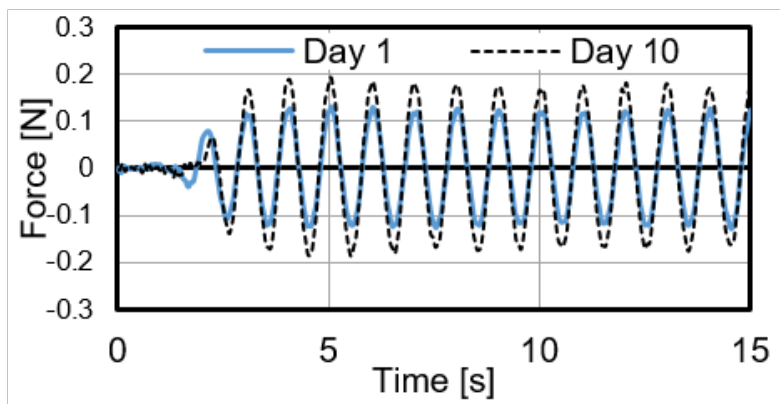

(a)

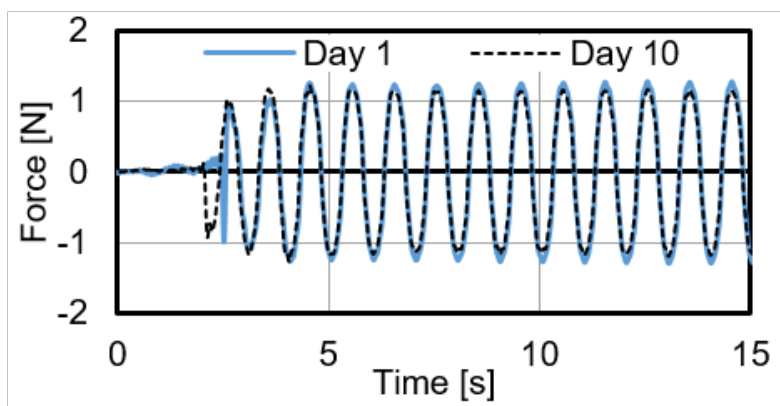

(b)

Figure 8. Time variation of the damping force of (a) the MR fluid damper and (b) MR grease damper in the OFF-state with the current of 0 A when starting to move, just after stirring, and 9 days after the static state.

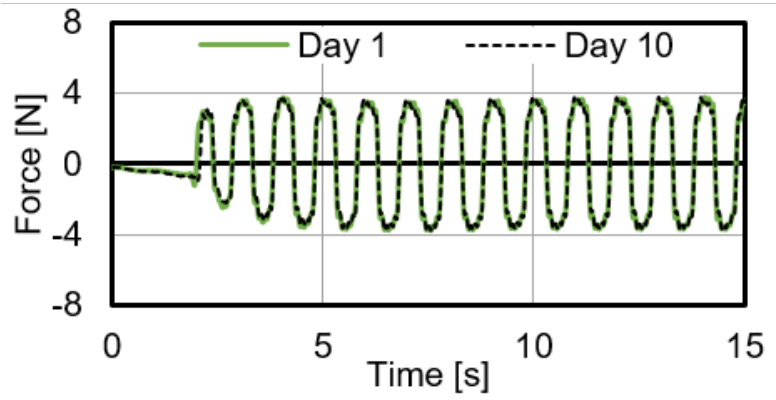

(a)

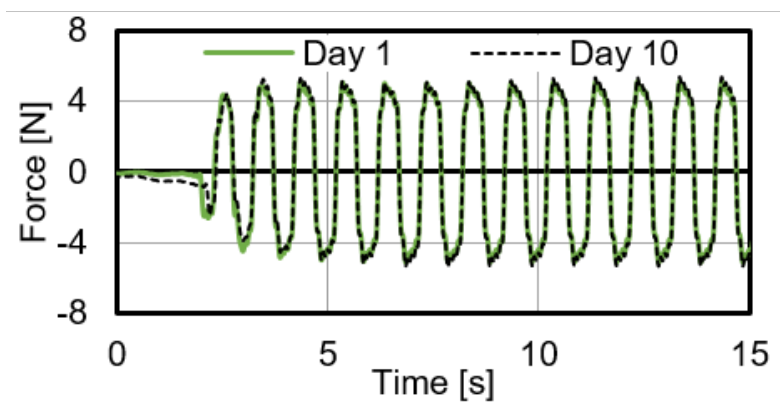

(b)

Figure 9. Time variation of the damping force of (a) the MR fluid damper and (b) MR grease damper in the ON-state with the current of $4 \mathrm{~A}$ when starting to move, just after stirring, and 9 days after the static state. 
Table 5. Changes in the RMS damping force of the MR dampers when starting to move on days 1-10 after the static state.

\begin{tabular}{ccccccc}
\hline & \multicolumn{3}{c}{ MR fluid damper } & \multicolumn{3}{c}{ MR grease damper } \\
\hline & Day 1 & Day 10 & Change & Day 1 & Day 10 & Change \\
& {$[\mathrm{N}]$} & {$[\mathrm{N}]$} & {$[\%]$} & {$[\mathrm{N}]$} & {$[\mathrm{N}]$} & {$[\%]$} \\
\hline OFF (0 A) & 0.09 & 0.12 & 41.6 & 0.96 & 0.89 & -6.8 \\
ON (4 A) & 3.08 & 3.06 & -0.7 & 4.08 & 4.25 & 4.2 \\
\hline
\end{tabular}

\section{Structural vibration suppression test}

To investigate the semi-active vibration suppression performance of the shear-type MR grease damper, the damper was applied to the vibration suppression of a structure. A small single-degree-of-freedom model structure equipped with the damper was subjected to actual seismic excitation (Figure 10). By evaluating the vibration transmissibility over a frequency range including the natural frequency, it was examined whether the damper could perform well regarding the vibration suppression of the structure.

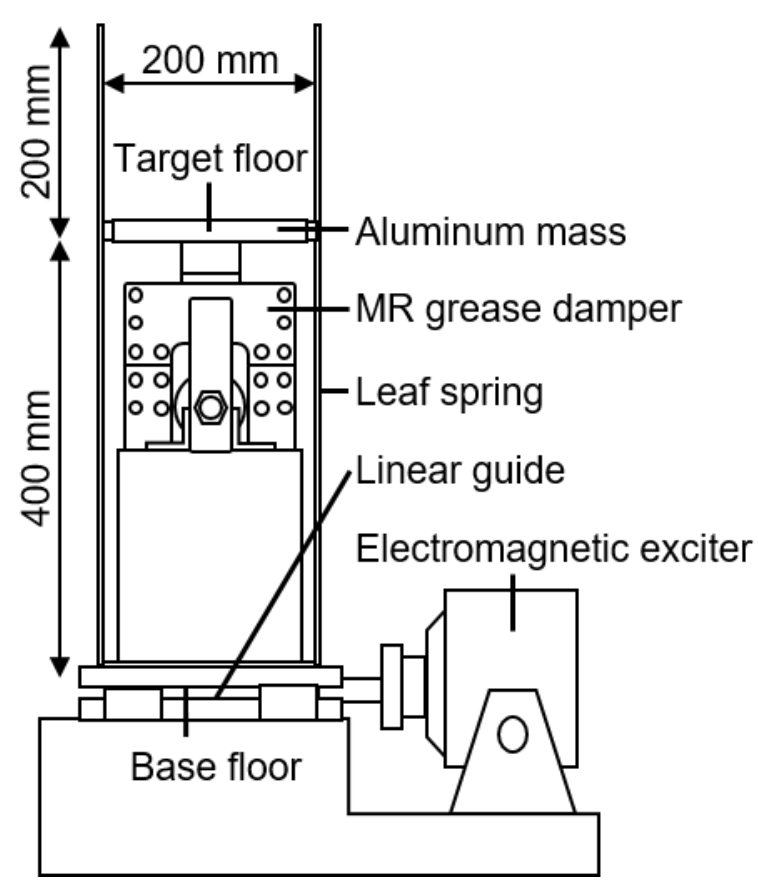

Figure 10. Schematic diagram of the small structure equipped with the shear-type MR grease damper.

\subsection{Methods}

The semi-active vibration suppression experiment comprises three procedures: base excitation, displacement measurement, and semi-active current control (Figure 11). First, the base floor of the structure was excited by an electromagnetic exciter using a seismic waveform transmitted from a controller. Then, the displacement response and input were measured by laser displacement sensors on the target and base floors, respectively. Finally, control currents calculated in the controller were supplied from a bipolar power supply to the electromagnetic coil in the MR damper. The current value was determined based on a control law and was updated every 5 ms. To compare the performance of the semi-active control with that of the passive states, this experiment was also conducted with 
constant currents. The currents of 0 and $4 \mathrm{~A}$ were supplied in both the OFF and ON states, respectively (Table 6). The winding number of the coil was determined considering the force magnitude and response time of the damper. In the case of 125 turns, the damping force in the ON-state was large enough to fix the upper and base floors at a natural frequency of $2.5 \mathrm{~Hz}$. The response time with the coil was fast enough that the supplied current coincided with the theoretical value of the controller.

Subsequently, the excitation input of the actual seismic wave and the control law of the skyhook-based control were described as important conditions to demonstrate that the shear-type MR grease damper can suppress vibrations by significantly changing the damping characteristics against the base motion of an earthquake.

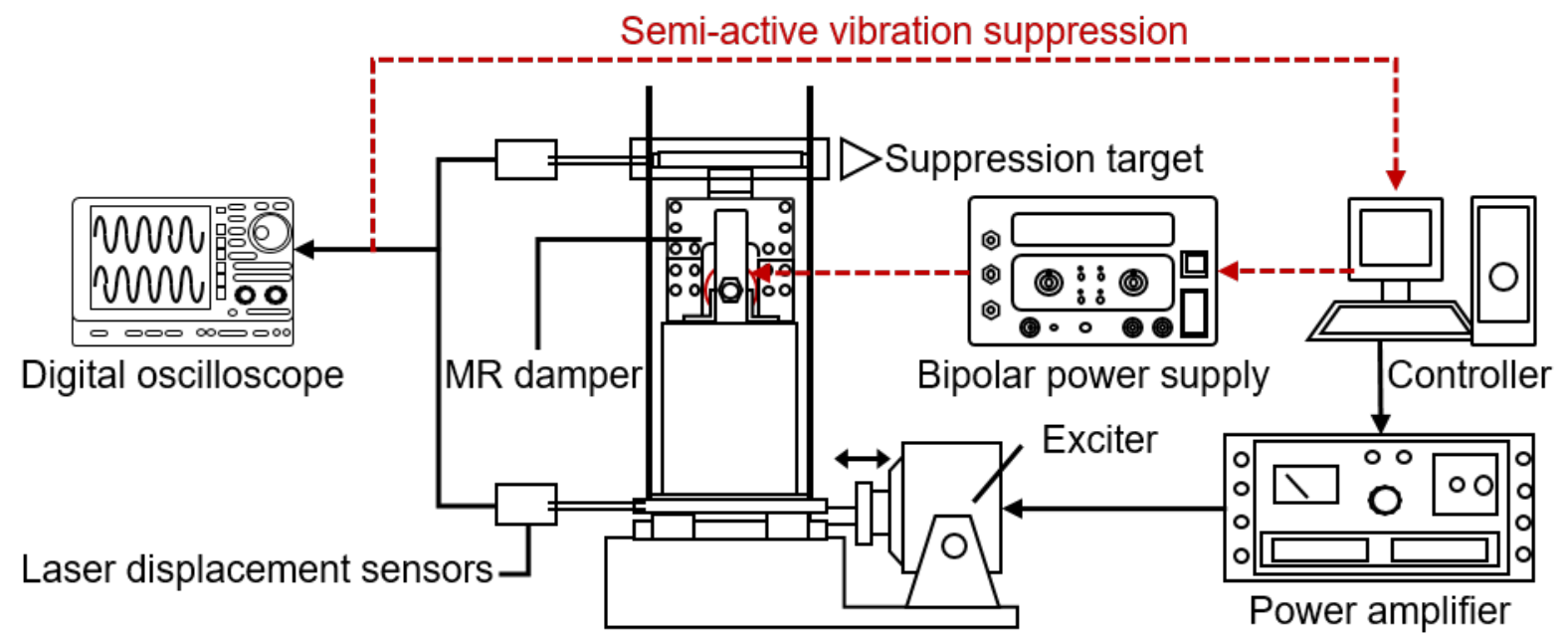

Figure 11. Schematic diagram of the experimental setup for the structural vibration suppression test.

Table 6. Experimental conditions for the structural vibration suppression test.

\begin{tabular}{cc}
\hline Current for passive & $0 \mathrm{~A}(\mathrm{OFF}), 4 \mathrm{~A}(\mathrm{ON})$ \\
Current for semi-active & $0-4 \mathrm{~A}$ (Variable) \\
Excitation input & Actual seismic wave \\
Displacement amplitude & $4.8 \mathrm{~mm}$ \\
of the base floor & $2.0-10.0 \mathrm{~Hz}$ \\
Dominant frequency & $\mathrm{ON}-\mathrm{OFF}$ control \\
Conventional control & Skyhook-based control \\
Proposed control & 125 turns \\
Winding number of the coil & $40^{\circ} \mathrm{C}$ \\
\hline
\end{tabular}

\section{Excitation input}

The small structure was subjected to an actual seismic wave. The recorded data during the 2011 earthquake that took place off the Pacific coast of Tohoku were used. The excitation input was the North-South component of the displacement observed in Namie Town, which was severely damaged by that earthquake. Its amplitude was scaled to a maximum of $4.8 \mathrm{~mm}$ considering the limitations of the displacement sensors and exciter (Figure 12). The frequency was modified so that the frequency of the original data became 10 times higher to examine whether the 
damper can provide high suppression performance not only around the natural frequency but also in the highfrequency range. The dominant frequency of the modified displacement was widespread from 2 to $10 \mathrm{~Hz}$ (Figure 13). Note that the frequency components lower than $2.0 \mathrm{~Hz}$ in the input data were removed by a low-pass filter as the exciter cannot work well at frequencies below $2.0 \mathrm{~Hz}$.

This excitation input was suitable for demonstrating the advantages of semi-active vibration suppression. Conventional passive vibration suppression methods cannot suppress the vibration caused by such inputs, as they have a wide range of dominant frequencies. In the passive states, the suppression performance degraded because of the fixed damping ratios. For example, when the damping ratio was always high, the response at high frequencies could not be suppressed. However, the response at low frequencies, including the natural frequency, could be successfully suppressed. Nevertheless, semi-active vibration methods can provide appropriate damping ratios according to the dominant frequency. The strategy of realizing the damping ratio variation with the vibration state is described, as shown below.

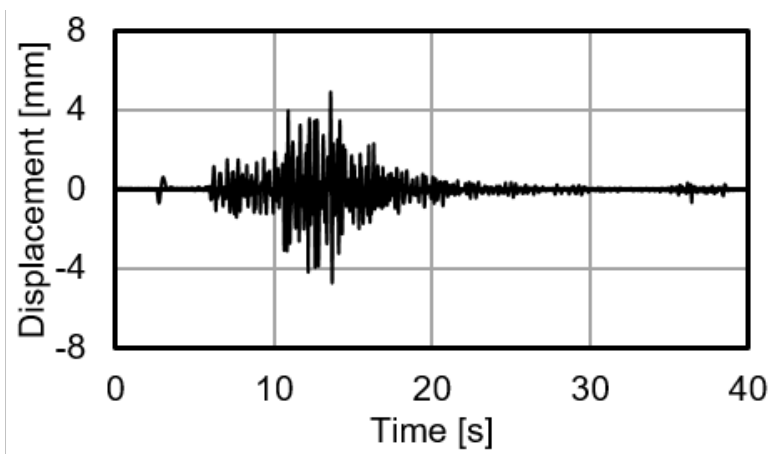

Figure 12. Time series of the input displacement measured on the base floor.

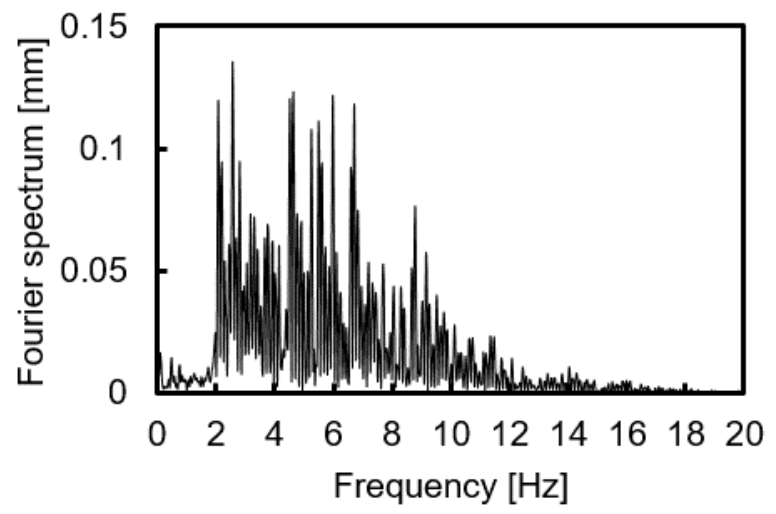

Figure 13. Fourier spectrum of the input displacement. 


\section{Control law}

To achieve high-performance vibration suppression, an appropriate control law for dampers should be used. The advantages of the shear-type MR grease damper are its high dynamic range and continuous controllability. In this study, a semi-active control law based on the skyhook damper scheme (Karnopp et al., 1974) was applied to make use of these advantages. The skyhook damper scheme proposed that the vibrations of single-degree-of-freedom systems can be successfully suppressed over an entire frequency range by exerting a damping force to reduce the absolute velocity of the suppression target. The desired damping force $F_{d}$ can be expressed as

$$
F_{d}=c_{s k y} \dot{x}
$$

where $\dot{x}$ denotes the target velocity. However, $F_{d}$ could not be achieved in this experiment as the damper between the upper and base floors generated forces to reduce the relative velocity $\dot{x}-\dot{x}_{0}$, where $\dot{x}_{0}$ is the base velocity. In this study, a skyhook-based control was proposed to generate the desired force within the achievable range. By controlling the current to the electromagnetic coil in the damper, the damping force was varied according to the following equation.

$$
F=\left\{\begin{array}{l}
F\left(I_{c}\right), \dot{x}\left(\dot{x}-\dot{x}_{0}\right)>0 \\
F_{O F F}, \dot{x}\left(\dot{x}-\dot{x}_{0}\right) \leq 0
\end{array}\right.
$$

When the desired and actual forces were in the same direction, the damping force was varied with the vibration state of the structure by the control current $I_{C}$. When they were opposite, the force was minimized by reducing the current to $0 \mathrm{~A}$ so as not to degrade the response.

To achieve the desired force $F_{d}$, the control current $I_{c}$ was determined by the target velocity $\dot{x}$, relative velocity $\dot{x}-\dot{x}_{0}$, and the parameters representing the relationship between the force, damper velocity, and current of the shear-type MR grease damper. In the structure, the damper velocity corresponded to the relative velocity between the floors, $\dot{x}-\dot{x}_{0}$. First, the force was approximated as a linear function of the damper velocity at each current (Figure 14). The magnitude of the damping force at the current of $I, F(I)$, could be expressed as

$$
F(I)=a(I) v+b(I),
$$

where $v$ denotes the relative velocity magnitude, $\left|\dot{x}-\dot{x}_{0}\right|$. Then, the gradients $a(I)$ and intercepts $b(I)$ were approximated as quadratic functions of the current (Figure 15). The force could be expressed as

$$
F(I)=\left(a_{2} I^{2}+a_{1} I+a_{0}\right) v+\left(b_{2} I^{2}+b_{1} I+b_{0}\right) .
$$

Using the damping coefficient $c$ (Figure 14), $F(I)$ could be also expressed as

$$
F(I)=c v+b_{0}
$$

From Eq. (4) and Eq. (5), the control current $I_{c}$ could be expressed as

$$
I_{c}=\frac{-\left(a_{1} v+b_{1}\right)+\sqrt{\left(a_{1} v+b_{1}\right)^{2}-4\left(a_{2} v+b_{2}\right)\left(a_{0}-c\right) v}}{2\left(a_{2} v+b_{2}\right)} .
$$

By applying Eq. (6) as $I_{c}$ in Eq. (2), the damping force proportional to the relative velocity could be achieved. In this study, to generate the desired damping force proportional to the absolute velocity instead of the relative velocity, the damping coefficient $c$ was updated using an equation expressed as

$$
c=c_{d} \frac{\dot{x}}{\dot{x}-\dot{x}_{0}}
$$

where $c_{d}$ denotes the proportional gain. Eq. (7) was proposed in a previous study as "continuous skyhook control" 
(Liu et al., 2005). By ignoring the intercept $b_{0}$, the actual damping force brought by Eq. (7) could be expressed using the following equation.

$$
F\left(I_{c}\right) \cong c_{d} \frac{\dot{x}}{\dot{x}-\dot{x}_{0}}\left(\dot{x}-\dot{x}_{0}\right)=c_{d} \dot{x}
$$

The damping force proportional to the target velocity could be achieved. Finally, the proposed skyhook-based control could be formulated as

$$
I=\left\{\begin{array}{l}
I_{c}, \dot{x}\left(\dot{x}-\dot{x}_{0}\right)>0 \\
0, \dot{x}\left(\dot{x}-\dot{x}_{0}\right) \leq 0
\end{array} .\right.
$$

In the following subsection, the results of the proposed skyhook-based control were compared with those of ON-OFF control, which has been widely used in conventional systems (Hu et al., 2017; Liu et al., 2005). A constant current of 4 A was supplied as $I_{c}$ in the case of the ON-OFF control.

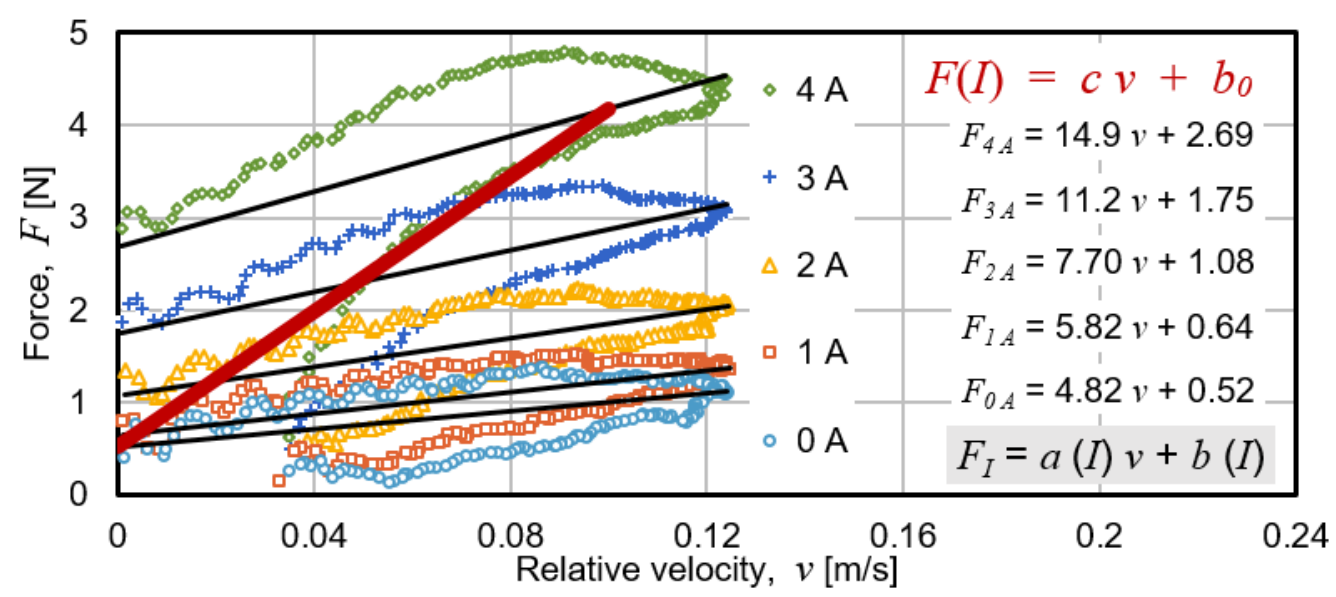

Figure 14. Approximation of the damping force as linear functions of the damper velocity at each current. The currentdependent gradients and intercepts were obtained at each current.

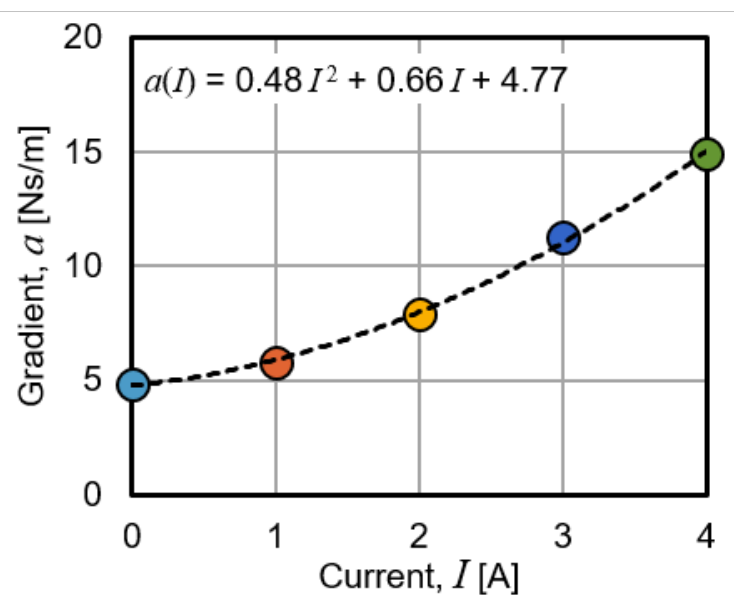

(a)

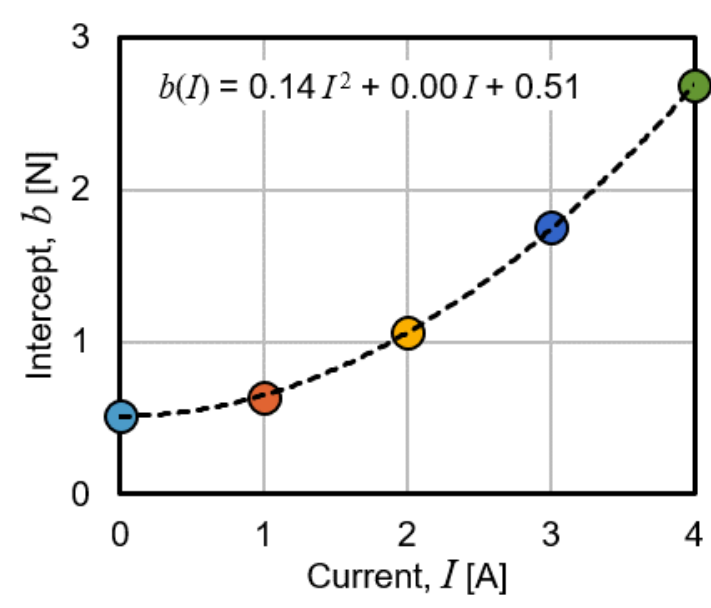

(b)

Figure 15. (a) Gradient and (b) intercept approximated as quadratic functions of a current. 


\subsection{Results and discussion}

The displacement responses in the OFF and ON states and with the proposed control were different from each other (Figure 16). The proposed skyhook-based control provided the highest performance in terms of reducing the maximum displacement response, which is defined as half the peak-to-peak amplitude (Figure 17). Its response reduction rate compared with the OFF-state was $24.1 \%$, whereas the ON-state and conventional ON-OFF control only achieved $9.1 \%$ and $7.5 \%$, respectively. Similarly, the proposed control achieved the highest response reduction rate of the RMS displacement (Table 7).

In the frequency domain, when the structural vibration was passively suppressed, there was a typical trade-off between the vibration suppression performance at low frequencies and that at high frequencies (Figure 18). The high damping ratio in the $\mathrm{ON}$-state was more effective in suppressing vibrations around the natural frequency of $2.5 \mathrm{~Hz}$, whereas the low damping ratio in the OFF-state provided a higher suppression performance in the highfrequency range. Such a trade-off was overcome by varying the damping ratio based on the vibration state using the proposed skyhook-based control. Around the natural frequency and in the high-frequency range, the displacement transmissibility with the proposed control was reduced to the same level as in the ON and OFF states, respectively.

In conventional studies, the "continuous skyhook control" effect on the vibration suppression performance has only been demonstrated using numerical simulations. Experimental demonstrations have not been performed as there were few dampers with a high dynamic range enough to significantly change the damping coefficient. However, the shear-type MR grease damper in this study succeeded in changing the damping coefficient $c$ over a wide range because of its high dynamic range. The current supplied in the experiment almost coincided with the theoretical one so that the damping coefficient $c$ could be changed to the desired value without time delay (Figure 19). The obtained frequency response indicates that the damper could successfully suppress vibrations not only around the natural frequency but also in the high-frequency range by varying the current to update the damping coefficient $c$ based on absolute and relative velocities.

These results demonstrate that the shear-type MR grease damper can suppress seismic response over a wide frequency range by applying skyhook-based control, which can take full advantage of high dynamic ranges, even when the excitation input has a wide range of dominant frequencies. 


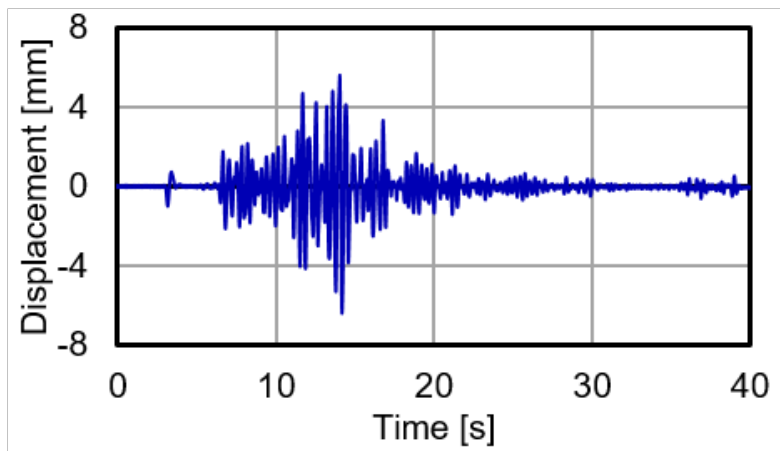

(a)

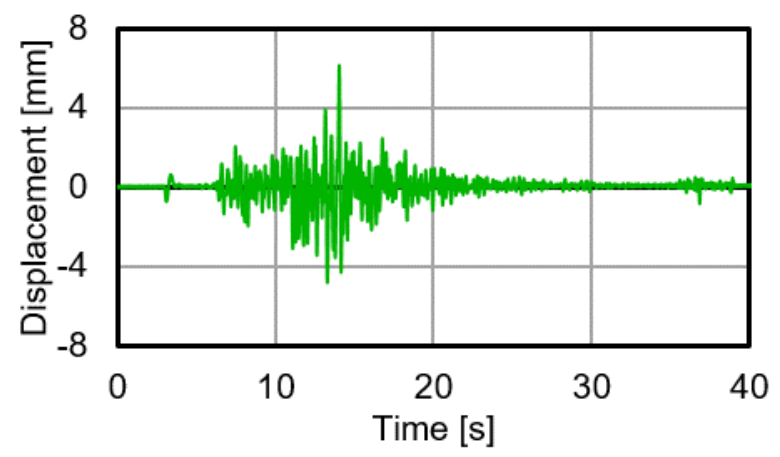

(b)

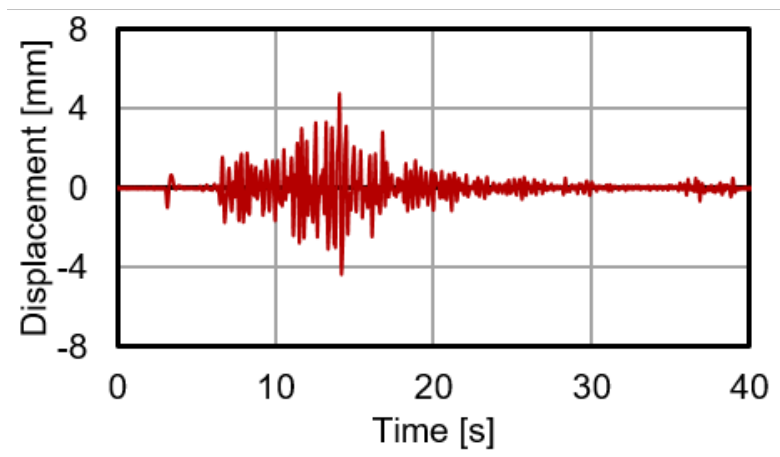

(c)

Figure 16. Time series of the displacement responses (a) in the OFF-state, (b) ON-state, and (c) with the proposed skyhook-based control.

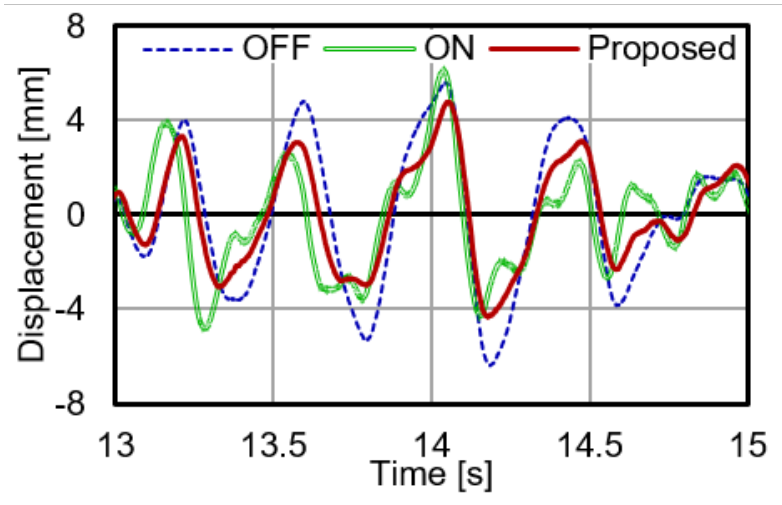

Figure 17. Enlarged time series of the displacement responses around their peaks. 
Table 7. Response reduction rates compared with the OFF-state.

\begin{tabular}{ccc}
\hline & Max [\%] & RMS [\%] \\
\hline OFF & 0 & 0 \\
ON & 9.1 & 25.6 \\
ON-OFF & 7.5 & 15.6 \\
Skyhook-based & 24.1 & 26.7 \\
\hline
\end{tabular}

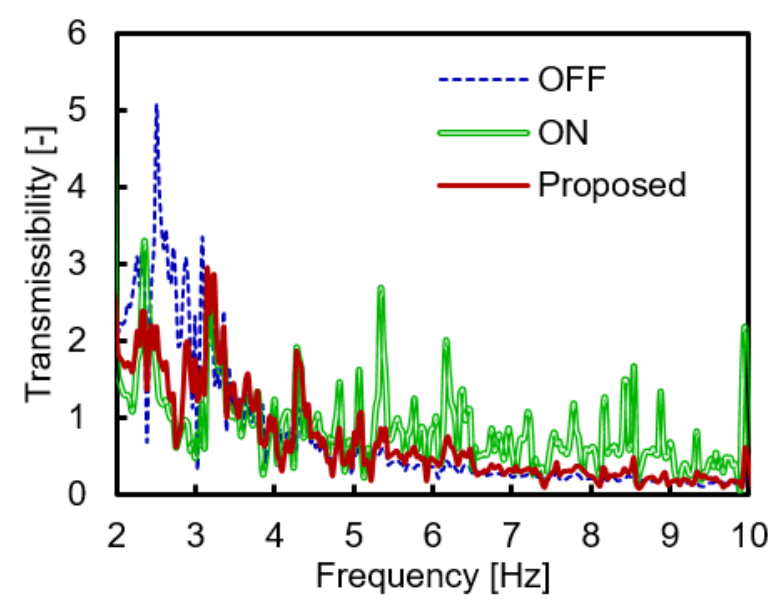

Figure 18. Frequency response of the structure equipped with the MR grease damper under seismic excitation.

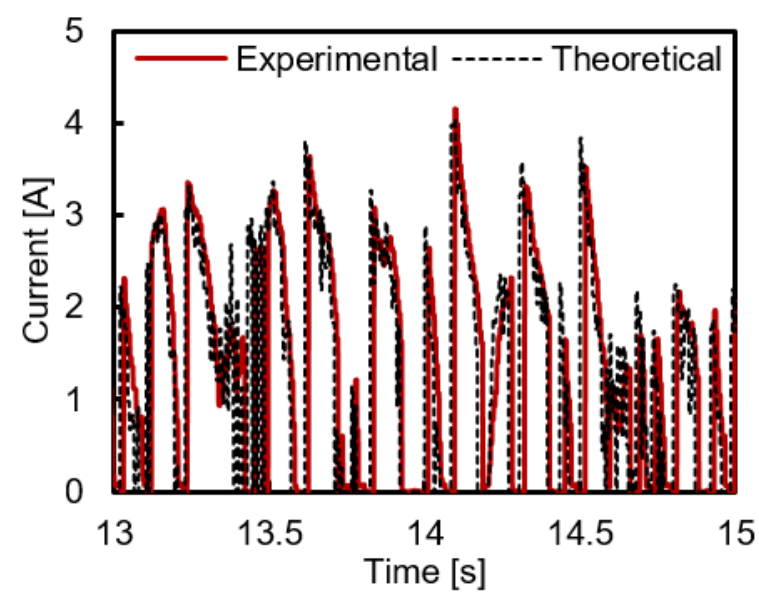

Figure 19. Enlarged time series of the current with the proposed skyhook-based control. 


\section{Conclusion}

This study experimentally investigated the dynamic range of a shear-type damper using MR grease. Also, the dispersion stability effect on the damping characteristics and the semi-active vibration suppression performance of a structure were investigated using the shear-type MR grease damper. First, a performance test of the damper was conducted to obtain its dynamic range as a ratio of the damping force at a current of $4 \mathrm{~A}$ in the $\mathrm{ON}$-state to the damping force at $0 \mathrm{~A}$ in the OFF-state. The results indicated that because of the advantage of combining MR grease and a shear-type damper, the damper showed an extremely high dynamic range reaching up to 157 times, which is much higher than that of conventional MR dampers. Second, another performance test of the damper was conducted to examine the time change of its damping characteristics. The results indicated that because of the high dispersion stability of MR grease, the damper had long-time reliability, thus keeping the damping characteristics in both the ON and OFF states even 9 days after the static state. However, the same damper type that used a conventional MR fluid changed the damping characteristics in the OFF-state. Finally, a vibration suppression test of a small single-degree-of-freedom model structure was conducted under seismic excitation to investigate the semi-active vibration suppression performance when using the shear-type MR grease damper. The results indicated that because of the high dynamic range of the damper realizing the desired damping coefficient in skyhook-based control, the damper had high vibration suppression ability that could ideally reduce the vibration response in a wide frequency range not only around the natural frequency but also in the frequency range higher than the natural frequency.

\section{References}

Ahamed R, Choi SB and Ferdaus MM (2018) A state of art on magneto-rheological materials and their potential applications. Journal of Intelligent Material Systems and Structures 29(10): 2051-2095.

Bahiuddin I, Wahab NA, Shapiai M, et al. (2019) Prediction of field-dependent rheological properties of magnetorheological grease using extreme learning machine method. Journal of Intelligent Material Systems and Structures 30(11): 1727-1742.

Changsheng Z (2006) Experimental investigation on the dynamic behavior of a disk type damper based on magnetorheological grease. Journal of Intelligent Material Systems and Structures 17(8-9): 793-799.

Dike SJ, Spencer Jr. BF, Sain MK, et al. (1998) An experimental study of MR dampers for seismic protection. Smart Materials and Structures 7: 693.

Hu Y, Chen MZQ and Sun Y (2017) Comfort-oriented vehicle suspension design with skyhook inerter configuration. Journal of Sound and Vibration 405(29): 34-47.

Karnopp D, Crosby MJ and Harwood RA (1974) Vibration control using semi-active force generators. Transactions of the American Society of Mechanical Engineers, Journal of Manufacturing Science and Engineering 96(2): 619-626.

Kavicoglu BM, Gordaninejad F and Wang X (2013) Study of a magnetorheological grease clutch. Smart Materials and Structures 22: 125030.

Kimura H, Kondo S, Ogawa T, et al. (2013) Magneto-rheological grease composition. US20130123153A1 Patent. Kintz KA, Carlson JD, Beth CM, et al. (2003) Magnetorheological grease composition. US6547986B1 Patent.

Lee HJ, Jung HJ, Moon SJ, et al. (2010) Experimental investigation of MR damper-based semiactive control algorithms 
for full-scale five-story steel frame building. Journal of Intelligent Material Systems and Structures 21(10): 1025-1037. Liu Y, Waters TP, Brennan MJ (2005) A comparison of semi-active damping control strategies for vibration isolation of harmonic disturbances. Journal of Sound and Vibration 280(1-2): 21-39.

Machacek O, Kubik M, Strecker Z, et al. (2019) Design of a frictionless magnetorheological damper with a high dynamic force range. Advances in Mechanical Engineering 11(3): 1-8.

Mohamad N, Mazlan SA, Ubaidillah, et al. (2019) The field-dependent viscoelastic and transient responses of plate-like carbonyl iron particle based magnetorheological greases. Journal of Intelligent Material Systems and Structures 30(5): 788-797.

Park E, Min KW, Lee SK, et al. (2010) Real-time hybrid test on a semi-actively controlled building structure equipped with full-scale MR dampers. Journal of Intelligent Material Systems and Structures 21(18): 1831-1850.

Rabinow J (1948) The magnetic fluid clutch. Transaction of American Institute of Electrical Engineers 67(2): 13081315.

Rankin PJ, Horvath AT and Klingenberg DJ (1999) Magnetorheology in viscoplastic media. Rheologica Acta 38: 471477.

Shen Y, Golnaraghi MF and Heppler GR (2004) Experimental research and modeling of magnetorheological elastomers. Journal of Intelligent Material Systems and Structures 15(1): 27-35.

Shiraishi T, Miida Y, Sugiyama S, et al. (2011) Typical characteristics of magnetorheological grease and its application to a controllable damper. Transactions of the Japan Society of Mechanical Engineers, Series C 77(778): 2193-2200 (in Japanese).

Shiraishi T and Misaki H (2016) Vibration control by a shear type semi-active damper using magnetorheological grease. Journal of Physics: Conference Series 744: 012012.

Shiraishi T and Sugiyama T (2015) Development of a shear type controllable damper with magnetorheological grease. Bulletin of the Japan Society of Mechanical Engineers, Mechanical Engineering Journal 2(3) : 14-00551.

Tu JW, Liu J, Qu WL, et al. (2011) Design and fabrication of 500-kN large-scale MR damper. Journal of Intelligent Material Systems and Structures 22(5): 475-487.

Wang H, Li Y, Zhang G, et al. (2019) Effect of temperature on rheological properties of lithium-based magnetorheological grease. Smart Materials and Structures 28: 035002.

Xu Y, Gong X, Xuan S, et al. (2011) A high-performance magnetorheological material: preparation, characterization and magnetic-machanic coupling properties. Soft Matter 7(11): 5246-5254.

Yang G, Spencer Jr. BF, Carlson JD, et al. (2002) Large-scale MR fluid dampers: modeling and dynamic performance considerations. Engineering Structures 24(3): 309-323.

Yao GZ, Yap FF, Chen G, et al. (2002) MR damper and its application for semi-active control of vehicle suspension system. Mechatronics 12(7): 963-973. 